\title{
Diseño de una oreja electrónica para caracterización de auriculares de audiometría
}

\author{
Mauro Gabriel Bravo Barranco ${ }^{1}$, Víctor J. Toranzos ${ }^{2}$ y Oscar G. Lombardero ${ }^{3}$
}

\section{RESUMEN}

La necesidad de contar con un instrumento para evaluar la respuesta de un auricular empleado en estudios de audiometría, motivó el desarrollo del presente trabajo de final de graduación, que consistió en el diseño y construcción de una oreja electrónica. Este dispositivo permite la medición de la presión sonora que se genera a partir de un auricular, permitiendo la caracterización del mismo, con énfasis en aquellos modelos que se emplean para estudios de audiometría. Este prototipo fue desarrollado en el Grupo de Ingeniería en Rehabilitación (GRIER) de la FACENA UNNE, y fue contrastado con un equipo comercial del Gabinete de Fonoaudiología del Instituto Hellen Keller de la ciudad de Corrientes, obteniéndose resultados muy satisfactorios.

\section{INTRODUCCION \\ Audiometría Tonal Liminar}

Un estímulo sonoro puede efectuarse en un sujeto por caminos diferentes: por vía aérea o por vía ósea [1]. En la conducción aérea, el estímulo sonoro llega al oído de la persona a través del conducto auditivo externo. El sonido presentado así se propaga a través del mismo, continua por el oído medio y alcanza el oído interno donde se produce la transducción del estímulo a un biopotencial. La audiometría tonal liminar es una prueba funcional audiológica complemen-taria, que determina el umbral auditivo aéreo y óseo (entre las frecuencias de 125 y $8000 \mathrm{~Hz}$ ), así como también permite diagnosticar y clasificar topográficamente el tipo de hipoacusia presente en dicha persona, aunque de todas maneras sigue siendo una prueba subjetiva.

Cualquier método de evaluación auditiva debe realizarse en un ambiente silencioso, normalmente en cabinas audiométricas de tipo anecoicas. Se utiliza particularmente un Audiómetro, que es un instrumento generador de sonidos puros (tonos), para un rango de frecuencias comprendidas entre 125 y $8.000 \mathrm{~Hz}$. El control de la intensidad de la emisión de dichos tonos se establece en decibeles HL.

1,2,3 GRIER Grupo de Ingeniería en Rehabilitación.FACENA UNNE.osguilom@yahoo.com.ar, victoranzos@gmail.com, 
El audiómetro cuenta con un juego de auriculares especiales que permite estudiar la vía aérea, así como para realizar el enmascaramiento. También dispone de un vibrador, que se utiliza para ser aplicado firmemente a la superficie craneal, generalmente a la apófisis mastoides, y así permitir la emisión de sonidos transmitidos por vía ósea.

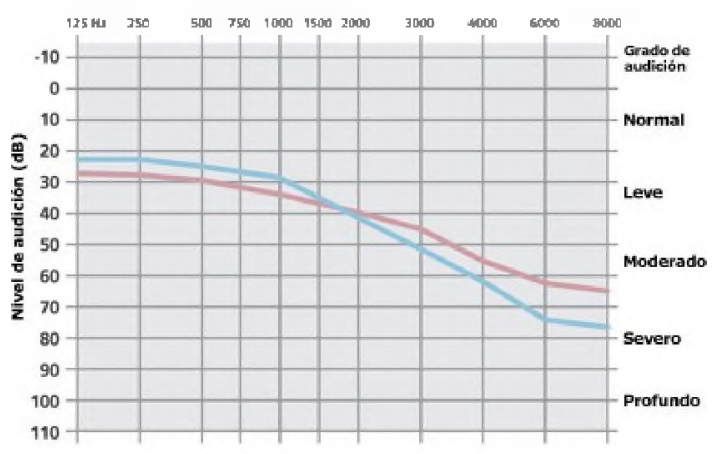

Fig. 1 Audiograma (con pérdida de audición)

En la Figura 1 se muestra un Audiograma donde sobre el eje de las ordenadas, están las intensidades de las señales senoidales en $\mathrm{dB}$ que reproduce el auricular y que estimulan los oídos de la persona bajo estudio. E1 rango en intensidad abarca desde $0 \mathrm{~dB}$ (algunos desde $-10 \mathrm{~dB}$ ) hasta $120 \mathrm{~dB}$, distribuidos en el audiograma de 10 en 10 .

\section{Presión sonora}

El sonido puede considerarse como una sucesión de ondas de compresión seguidas por ondas de descompresión que se propagan por el aire a una velocidad de $345 \mathrm{~m} / \mathrm{s}$. [2]. Sin embargo, al observar desde una posición fija, la presión atmosférica aumenta y disminuye periódicamente, conforme pasan por el lugar las sucesivas perturbaciones. $\mathrm{La}$ unidad adoptada internacional-mente para la presión es el Pascal, abreviada Pa. Expresada en esta unidad, la presión atmosférica es del orden de $1.000 \mathrm{hPa}$, (hectopascal).

Ahora bien, los aumentos y las disminuciones de presión debidas a las ondas sonoras son realmente muy pequeños comparados con este valor de presión atmosférica. Los sonidos más intensos que se perciben como tales (después de eso se perciben como dolor) implican un aumento de unos $20 \mathrm{~Pa}$. Para distinguir este incremento de la presión atmosférica en ausencia de sonido, se lo denomina presión sonora, abreviada $\mathrm{p}$. Las presiones sonoras audibles varían entre 0,00002 $\mathrm{Pa}$ y $20 \mathrm{~Pa}$. El valor más pequeño, también expresado como $20 \mu \mathrm{Pa}$, se denomina umbral auditivo.

\section{Nivel de presión sonora}

Para el rango de los sonidos audibles, la presión sonora varía entre los $20 \mu \mathrm{Pa}$ hasta los $20 \mathrm{~Pa}$. Estas cifras son poco prácticas de manejar, por lo cual se introduce otra escala en $\mathrm{dB}$ que comprime este rango. Para esto se define primero una presión de referencia Pref que es la mínima presión sonora audible (correspondiente al sonido más débil que se puede escuchar), o sea

$$
P_{\text {Ref }}=20[\mu P]
$$

Entonces se define el nivel de presión sonora, NPS (o SPL Sound Pressure Level), mediante la siguiente fórmula:

$$
N P S=20 * \log \left(\frac{P}{\mathrm{P}_{\mathrm{ref}}}\right)[d B]
$$


Donde $\mathrm{P}$ es la presión sonora, y log el logaritmo en base 10. La unidad dB HL no es lo mismo que la dB SPL, ya que la primera está relacionada a cómo interpreta el oído promedio las variaciones de presión y eso depende de la frecuencia. En cambio, la segunda es la variación de presión respecto a $20 \mu \mathrm{Pa}$, evaluado en una escala logarítmica.

Esta unidad se define según así porque los seres humanos, en promedio, recién comienzan a escuchar un tono de $250 \mathrm{~Hz}$ cuando éste se fija en $26,5 \mathrm{~dB}$ SPL y por ello se establece como $0 \mathrm{~dB}$ HL para la frecuencia de $250 \mathrm{~Hz}$ [9]. Luego, para $500 \mathrm{~Hz}$ la mayoría de los humanos comienzan a percibir el tono a los $13,5 \mathrm{~dB}$, al cual se le asigna el valor de 0 dB HL para esa frecuencia. Exactamente de la misma forma se procede para establecer el nivel de referencia en dB HL para todas las frecuencias utilizadas en audiometría, permitiendo la construcción de la Tabla I.

Tabla I Conversión de dB a dB HL

\begin{tabular}{|r|c|c|}
\hline $\mathbf{f}(\mathbf{H z})$ & Nivel SPL(dB) & Nivel HL (dB) \\
\hline 125 & 47,5 & 0 \\
\hline 250 & 26,5 & 0 \\
\hline 500 & 13,5 & 0 \\
\hline 1000 & 7,5 & 0 \\
\hline 2000 & 11 & 0 \\
\hline 4000 & 10,5 & 0 \\
\hline 8000 & 13 & 0 \\
\hline
\end{tabular}

\section{Sonoridad}

La sensación de sonoridad, es decir de intensidad de un sonido, está, en principio, relacionada con su amplitud. Sin embargo, la rela- ción no es tan directa como la que existe entre la frecuencia y la altura. De hecho, la sonoridad resulta fuertemente dependiente no sólo de la amplitud sino también de la frecuencia. Así, a igualdad de frecuencias podemos afirmar que un sonido de mayor amplitud es más sonoro. En la Figura 3 se muestran dos ondas de 200 $\mathrm{Hz}$, de las cuales la de mayor amplitud es más sonora. Pero si aumentamos la frecuencia del sonido de menor amplitud, éste puede llegar a percibirse como más sonoro. En el ejemplo de la Figura 4, el sonido de menor amplitud se lleva a $600 \mathrm{~Hz}$, percibiéndose ahora con mayor sonoridad.

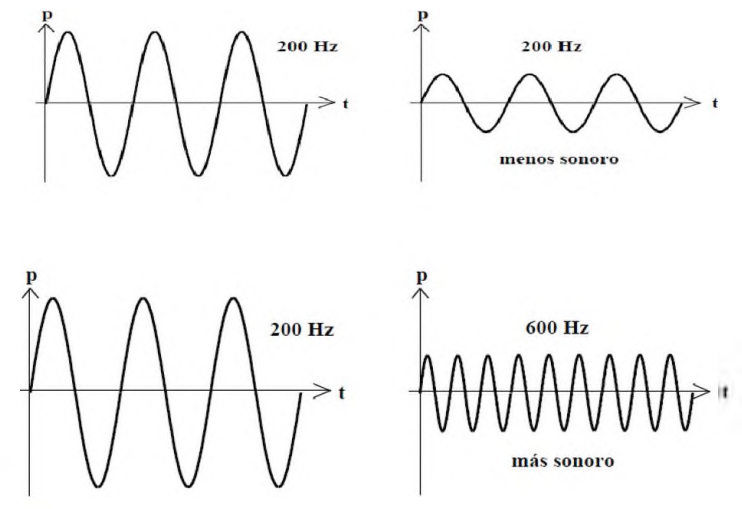

Fig. 2. Tipos de ondas y sus sonoridades

\section{Micrófono}

Son transductores capaces de convertir señal sonora en señal eléctrica. A los fines de conectar un micrófono, es importante conocer cuánta tensión produce ante una dada presión sonora, o sea la curva de respuesta. En la Figura 3 se muestran las formas de onda de la presión y la tensión. La sensibilidad de un micrófono puede definirse como el cociente entre la tensión producida y la presión que le da origen, es decir, 


$$
S=\frac{V}{P}
$$

Como la unidad de la presión es el Pascal, (que equivale a 10 millonésimas de la presión atmosférica), la sensibilidad de un micrófono se expresa en Volts por Pascal (V/Pa). Otra manera muy difundida para expresar la sensibilidad es en $\mathrm{dB}$ referidos a $1 \mathrm{~V} / \mathrm{Pa}$. En ese caso, llamando sensibilidad de referencia, Sref, a $1 \mathrm{~V} / \mathrm{Pa}$, se obtiene con esta fórmula:

$$
S_{d B}=20 * \log \left(\frac{S}{S_{r e f}}\right)
$$

Por ejemplo, un micrófono que ante una presión sonora de $0,2 \mathrm{~Pa}$ desarrolla una tensión de $1 \mathrm{mV}$, tendrá una sensibilidad de

$$
\mathrm{S}=\frac{0,001[\mathrm{~V}]}{0,2[\mathrm{~Pa}]}=0,005\left(\frac{\mathrm{V}}{\mathrm{pa}}\right)
$$

que en dB será

$$
S_{d B}=20 * \log \left(\frac{0,005}{1}\right)=-46[d B]
$$

\section{Ruido}

En los micrófonos hay dos mecanismos que generan ruido. El de ruido ambiental, que podría disminuirse a través de una regla de direccionalidad, y el ruido eléctrico, intrínseco al micrófono, y que podría reducirse diseñando el micrófono con muy baja impedancia (menor de $100 \Omega$ ). Este parámetro depende de la calidad del micrófono, por lo que podría conseguirse un nivel de ruido de $0,18 \mu \mathrm{V}$ aproximadamente. Por lo tanto, en la especificación técnica debe verificarse dicho nivel.

Hay dos maneras de vincular el ruido eléctrico. La primera consiste en asociarlo a un nivel de presión sonora equivalente, como por ejemplo $17 \mathrm{~dB}$. En otras palabras, se lo considera como un valor pequeño de tensión, que se adiciona a la respuesta hipotética de un micrófono sin ruido. Esta especificación sugiere cuál es el mínimo nivel sonoro para el cual tendrá sentido utilizar este micrófono. Si se desea registrar un sonido de menor nivel que el umbral de referencia, el ruido propio sería más intenso, con un deterioro de la calidad sonora, o bien un enmascaramiento de la señal original.

La segunda forma es a través del concepto de relación señal/ruido, expresada en $\mathrm{dB}$ :

$$
S / R=20 * \log \left(\frac{\text { signal }}{\text { noise }}\right)
$$

\section{Distorsión}

La distorsión es una medida de la deformación de la onda, mientras que el ruido es una señal independiente que se agrega a la señal. Cuando la señal es una onda senoidal pura, la distorsión se manifiesta como la aparición de una cierta cantidad de armónicos. Se define la distorsión total armónica (THD) como el cociente entre el valor eficaz de los armónicos generados por la distorsión y el valor eficaz de la fundamental, expresándose en porcentajes. Por ejemplo, si se excita un micrófono con un sonido senoidal, y como resultado produce una tensión de $5 \mathrm{mV}$ de primera armónica y 0,05 $\mathrm{mV}$ de las restantes armónicas, la distorsión 
tota

$$
\mathrm{TDH}=\frac{0,05[\mathrm{mV}]}{5,[\mathrm{mV}]}=1 \%
$$

Dado que el fenómeno de la distorsión se presenta normalmente para niveles altos de señal, la especificación está asociada con el máximo nivel de presión sonora que admite el micrófono. Una posible especificación podría ser:

THD: $1 \%$ a 125 dB NPS

\section{MATERIALESY METODOLOGÍA}

En la Fig. 3 se puede observar el diagrama en bloques del sistema.

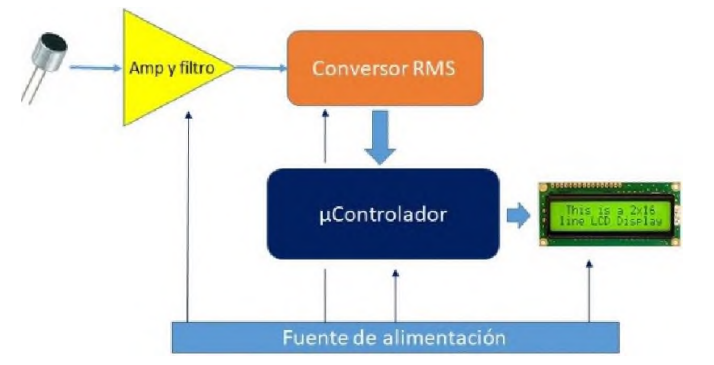

Fig. 3. Diagrama en bloques del sistema

El micrófono tipo Electret se ubicó en una cavidad equivalente a una oreja humana, y también se empleó una cabeza humana de dimensiones estándar utilizándose la técnica de la impresión 3D. El amplificador buffer fue necesario para desacoplar las impedancias. Se emplearon dos filtros, de los cuales el primero de 4to orden tipo Butterworth con $\mathrm{Fc}=12 \mathrm{KHz}$ y el segundo tipo Notch fijado a $50 \mathrm{~Hz}$ para filtrar ruido de línea. El módulo conversor RMS (AD736) realiza una conversión entre una señal de entrada dada, que podría tener cualquier forma y valor eficaz, y la representa a la salida como un valor de tensión continua equivalente al valor RMS.

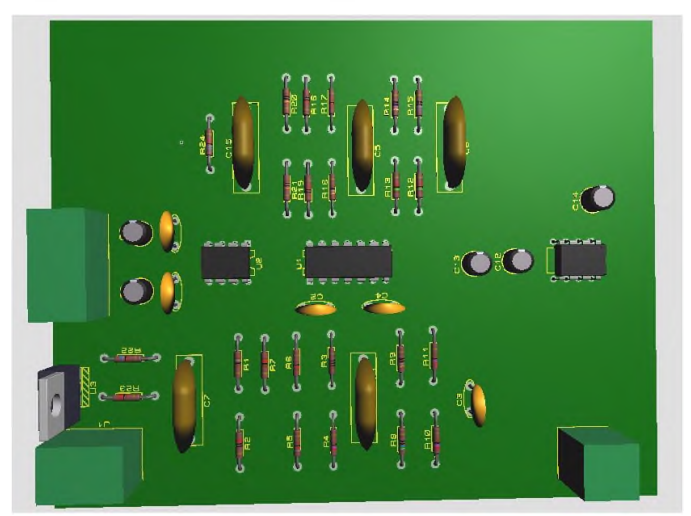

Fig. 4. Placa impresa de la oreja electrónica

\section{Calibración}

El objetivo fue determinar la relación entre la tensión de continua a la salida del conversor RMS y la presión sonora a la salida de los auriculares de un audiómetro comercial perteneciente al Instituto Hellen Keller, de la Ciudad de Corrientes. La disposición fue la que se muestra en la Figura 5.

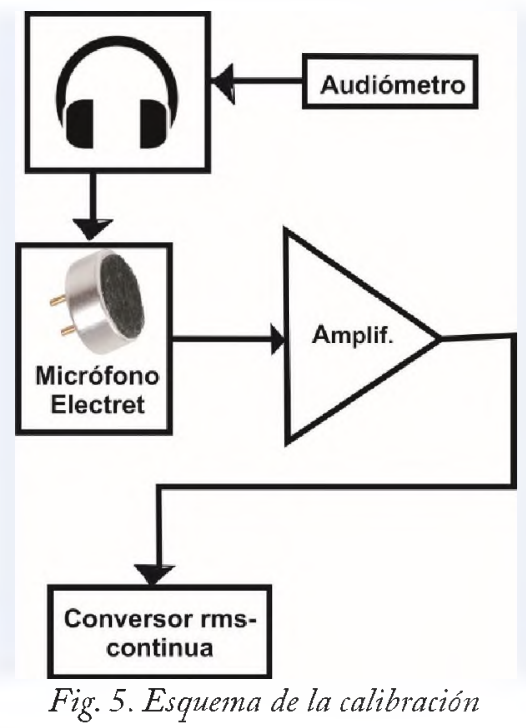


Luego de realizar un barrido monotonal entre 250 y $8000 \mathrm{~Hz}$, centradas en 250 , $500,1 \mathrm{KHz}, 2 \mathrm{KHz}, 4 \mathrm{KHz}$ y $8 \mathrm{KHz}$, se pudo establecer una relación lineal entre la presión sonora y la tensión de continua a la salida del conversor, para el rango de $80 \mathrm{~dB}$ HL y $100 \mathrm{~dB}$ HL.

\section{Repetitividad}

Se ensayó este parámetro en unos auriculares marca NOGANET con tonos descargados desde internet $(250 \mathrm{~Hz}, 500$ $\mathrm{Hz}, 1 \mathrm{Khz}, 2 \mathrm{Khz}, 4 \mathrm{Khz}$ y $8 \mathrm{Khz}$ ), a los cuales se le controló el volumen mediante dos programas ejecutados en simultáneo (El administrador de Sonido Realtek y el VLC Media Player). Se fijó el porcentaje de volumen de ambos programas en valores específicos a diferentes días de la semana. Los resultados figuran en la Fig. 6.

\begin{tabular}{|c|c|c|c|c|}
\hline \multicolumn{3}{|c|}{ Parémetros de control } & \multirow{2}{*}{$\begin{array}{c}23 / 10 / 2017 \\
\text { Oreja (dB } \\
\mathrm{HL})\end{array}$} & \multirow{2}{*}{$\begin{array}{c}25 / 11 / 2017 \\
\text { Oreja (dB } \\
H L)\end{array}$} \\
\hline $\begin{array}{c}F \\
(H z)\end{array}$ & $\begin{array}{l}\text { VLC } \\
(\%)\end{array}$ & $\begin{array}{c}\text { Compu } \\
(\%)\end{array}$ & & \\
\hline \multirow{3}{*}{250} & 70 & 50 & 86 & 86 \\
\hline & 70 & 70 & 91.7 & 91 \\
\hline & 91 & 70 & 97,5 & 97,67 \\
\hline \multirow{4}{*}{500} & 50 & 72 & 87,66 & 88 \\
\hline & 61 & 72 & 94,39 & 95 \\
\hline & 70 & 90 & 99,3 & 100 \\
\hline & 91 & 90 & 102,5 & 102 \\
\hline \multirow{3}{*}{$1 \mathrm{k}$} & 72 & 50 & 86,22 & 87 \\
\hline & 72 & 70 & 90,7 & 91 \\
\hline & 72 & 80 & 93 & 93 \\
\hline \multirow{4}{*}{$2 \mathrm{k}$} & 70 & 60 & 83,48 & 84,12 \\
\hline & 70 & 70 & 85,76 & 86 \\
\hline & 80 & 80 & 91,44 & 92 \\
\hline & 100 & 90 & 96 & 96 \\
\hline \multirow{3}{*}{$4 \mathrm{k}$} & 70 & 70 & 78,08 & 77 \\
\hline & 80 & 90 & 85,2 & 85 \\
\hline & 80 & 66 & 70,13 & 71 \\
\hline \multirow[t]{2}{*}{$8 k$} & 80 & 80 & 73,8 & 74 \\
\hline & 100 & 100 & 812 & 81 \\
\hline
\end{tabular}

Fig. 6. Veriffcación de la repetitividad

\section{Distorsión armónica total (TDH)}

Para determinar el TDH del sistema se inyectó un tono de $1 \mathrm{~Pa}$ a la frecuencia de $1 \mathrm{Khz}$ en los auriculares y se observó la respuesta de la Oreja Electrónica a la salida de la etapa del Amp. y Filtro. La respuesta que se obtuvo se observa en la Fig. 7 .

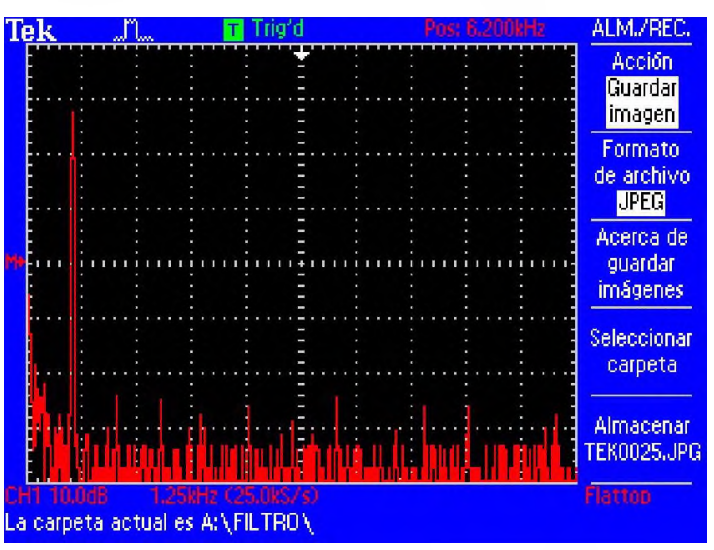

Fig. 7. Distorsión armónica generada por la oreja electrónica

\section{CONCLUSION}

Se diseñó e implementó una oreja electrónica que permite caracterizar un auricular comercial, especialmente aquellos que son utilizados en estudios de audiometría. También fue contrastado con un Audiómetro comercial Kamplex KD9.

Este instrumento mide la presión sonora a la salida de un auricular en un rango de 80 [dB HL] hasta 100 [dB HL]. E1 equipo no determina el TDH de los auriculares, por lo cual es importante determinar en qué magnitud los auriculares presentan este fenómeno. La exactitud obtenida por el instrumento fue de 2 [dB], lo cual es aceptable si se considera que los auriculares de 
audiometría presentan un error en su respuesta de $3 \mathrm{~dB}$ para valores inferiores a 2 Khz, y de $5 \mathrm{~dB}$ para superiores.

E1 TDH del instrumento diseñado fue menor al 2,5\%, valor aceptable dado que dicho valor es el TDH de un auricular típi- co de una audiometría (HDA 300).

Con ciertas modificaciones se podría utilizar el dispositivo para caracterizar estudios de grabación, cabinas sonoamortiguadas, así como también materiales acústicos con paneles fonoabsorbentes o distintos fonacs.

\section{BIBLIOGRAFÍA}

1. Pomajapon, Nathalie Beatriz. "Perfil Audiométrico de Usuarios con Tinnitus, atendidos en el Centro Ecuatoriano de Audición y Lenguaje (CEAL) de la Ciudad de Loja , durante Marzo-Agosto de 2015", Tesis previa para la obtención de título de médico general. Universidad Nacional de Loja. Año 2017

2. Federico Miyara. "Acústica y Sistemas de Sonido". Editorial de la Universidad de Rosario. Año 1999.

3. Alejandro Shroeder. "Medición de la Respuesta en frecuecnia de los auriculares". Universidad de Buenaventura. Bogota. Año: 2007

4. Ryouichi Nishimura, Member IEEE, Parham Mokhtari, Member IEEE, Hironori Takemoto, and Hiroaki Kato. "An Attempt to Calibrate Headphones for Reproduction of Sound Pressure at the Eardrum”. 2011.

5. Packman, Emilio. "Mediciones Eléctricas" (3o Edición). H.A.S.A. (Hispano América S.A.) - Argentina 1989.

6. Daniel Tehevenet. “Oreja Electrónica para controlar el ruido en unidades de cuidado intensivo". Año 2012.

7. Ernesto Accolti, Federico Miyara, Ezequiel Mignini. "Protocolo de calibración de auriculares audiométricos para su uso en investigación psicoacústica”. Año 2009.

\section{Referencia de Internet}

8. https://www.hear-it.org/es/Audiograma-3

9. http://audiologiaacademica.blogspot.com. ar/2014/09/audiometria-busqueda-de-umbrales.html

10.https://www.headphone.com/ 